\title{
Práticas Integrativas e Complementares na atenção básica em saúde: um estudo bibliométrico da produção brasileira
}

\author{
Integrative and Complementary Practices in basic health care: $a$ \\ bibliometric study of Brazilian production
}

Jordana Aguiar1, Lilia Aparecida Kanan', Anelise Viapiana Masiero1

DOI: 10.1590/0103-1104201912318

RESUMO As Práticas Integrativas e Complementares (PICs) são realidade no Sistema Único de Saúde (SUS) desde 2006, pela Política Nacional de Práticas Integrativas e Complementares (PNPIC). Este estudo objetivou conhecer as principais características da produção científica sobre PICs na atenção básica em saúde desenvolvida no Brasil durante os 10 primeiros anos de implementação da PNPIC, além de apontar os principais resultados constatados nesses estudos. O método foi sustentado pela bibliometria. Como resultado, destacam-se as pesquisas vinculadas à Universidade Federal de Santa Catarina (UFSC) e publicações do periódico 'Ciência e Saúde Coletiva'. O maior número de artigos foi publicado entre 2011 e 2014. Grande parte deles pretendeu analisar o contexto da implementação e do uso das PICs. Foram citadas, especialmente, fitoterapia, homeopatia e acupuntura. Como resultado do uso das PICs, encontraram-se: redução da medicalização; empoderamento e responsabilização dos usuários; redução da frequência de transtornos mentais comuns; baixo custo; ausência de efeitos colaterais; promoção de saúde. Entre os principais problemas tratados estão: transtornos mentais; relações sociais; psicossomáticos; insônia; doenças crônicas. As potencialidades e fragilidades citadas nos estudos merecem mais atenção por parte da academia e dos gestores da área da saúde.

PALAVRAS-CHAVE Atenção Primária à Saúde. Terapias complementares. Saúde pública.

ABSTRACT Integrative and Complementary Practices (PICs) are a reality in the Unified Health System (SUS) since 2006, by the National Policy on Integrative and Complementary Practices (PNPIC). This study aimed to know the main characteristics of scientific production on PICs in basic health care developed in Brazil during the first 10 years of PNPIC implementation, in addition to pointing out the main results observed in these studies. The method was supported by bibliometrics. As a result, the researches related to the Federal University of Santa Catarina (UFSC) and publications of the journal 'Ciência e Saúde Coletiva' stand out. The largest number of articles was published between 2011 and 2014. Most of them intended to analyze the context of implementation and use of PICs. Phytotherapy, homeopathy and acupuncture were the most mentioned. As a result of the use of PICs, it was founded: reduction of medicalization; empowerment and accountability of users; reduction of the frequency of common mental disorders; low cost; absence of side effects; health promotion. Among the main problems are: mental disorders; social relationships; psychosomatic; insomnia; chronic diseases. The potentialities and weaknesses cited in the studies deserve more attention from academia and managers of the health area. 


\section{Introdução}

Nos serviços de saúde, são recorrentes os indivíduos portadores de doenças crônicas e sofrimentos mentais, situações onde nem sempre o modelo biomédico é suficiente para a redução de sintomas e prevenção de agravos, tornando, assim, a inserção de Práticas Integrativas e Complementares (PICs) relevante para preencher essa carência'. Nesse contexto, as PICs representam um conjunto de recursos capazes de atuar nos diferentes aspectos da saúde, propiciando tanto a recuperação da saúde quanto a prevenção de doenças e agravos, sejam eles físicos ou mentais'. Elas se apresentam vantajosas por se tratar de métodos não medicamentosos, voltados ao autocuidado, que privilegiam a escuta acolhedora, o vínculo e a integração com o meio ambiente e a comunidade ${ }^{1}$. Os profissionais que exercem esse modelo de cuidado ofertam alternativas diferentes de promoção da saúde e renovam o entendimento atual, que tende especialmente à medicalização e aos procedimentos invasivos ${ }^{2}$.

As PICs representam uma perspectiva ampliada sobre o ser humano e o universo que o cerca, compreendem a integralidade da relação saúde-doença e consideram o sujeito dentro de uma dimensão global, ainda valorizando sua individualidade ${ }^{3}$.

Em sua primeira publicação, a Política Nacional de Práticas Integrativas e Complementares (PNPIC) incluía 5 práticas. Atualmente, encontram-se inseridos 29 tipos de práticas, que vão desde medicina tradicional chinesa até constelação familiar ${ }^{4}$.

Em razão da PNPIC fazer parte do sistema de saúde pública brasileiro há mais de uma década e sabendo que tais práticas, em geral, possuem boa aceitação e significativa efetividade, por meio deste estudo, pretendeu-se conhecer as características da produção científica brasileira sobre as PICs na atenção básica em saúde realizada nos primeiros 10 anos de implementação da Política Nacional que as trouxe para o Sistema Único de Saúde (SUS). Dessa forma, pretendeu-se colocar em perspectiva as principais características desses estudos, além de observar suas conclusões.
Estabeleceu-se como objetivo geral conhecer as características da produção brasileira sobre PICs na atenção básica. Especificamente, pretendeu-se (i) identificar as tendências de pesquisas brasileiras sobre PICs; (ii) avaliar a utilização das PICs na atenção básica; (iii) registrar os principais resultados das pesquisas empreendidas no Brasil a respeito das PICs.

\section{Percurso metodológico}

Este estudo foi sustentado pelo método bibliométrico, técnica quantitativa e estatística de medição ${ }^{5}$. Por meio desse método de pesquisa documental, buscou-se verificar a produção científica acerca de PICs desenvolvida no Brasil, entre os anos de 2006 e 2016.

O estudo assumiu caráter descritivo-exploratório. Para a pesquisa documental, foram consultadas as bases de dados: Literatura Latino-americana e do Caribe em Ciências da Saúde (Lilacs), Scientific Electronic Library Online (SciELO), Biblioteca Virtual em Saúde (BVS) e Índice Bibliográfico Español en Ciencias de la Salud (Ibecs), com o descritor 'Práticas Integrativas e Complementares', onde foram aplicados filtros coerentes com os critérios de inclusão e exclusão delimitados. Como critérios de inclusão para a seleção dos artigos, foram considerados: (a) o período de publicação entre 2006 e 2016; (b) artigos publicados no Brasil e nos idiomas português, inglês ou espanhol; (c) artigos em open access; (d) artigos que relacionem as PICs na atenção básica em saúde. Excluíram-se todas as publicações: (a) que não eram artigos; (b) que se encontravam fora do limite de tempo estabelecido (entre 2006 e 2016) para a busca; (c) que eram revisões bibliográficas e pesquisas documentais. Para selecionar os artigos, primeiramente, procedeu-se à leitura do título e do resumo; em seguida, os artigos pré-selecionados foram lidos na íntegra para avaliação da sua compatibilidade com os critérios de inclusão previamente estabelecidos para o estudo. 
O período de busca, de coleta e tratamento dos dados aconteceu entre abril e outubro de 2018. Os artigos publicados em 2017 não foram inseridos no estudo em razão de se haver privilegiado o período de 10 anos de implementação da PNPIC.

As etapas da seleção estão expressas na figura 1, a seguir.

Figura 1. Etapas do processo de seleção dos artigos

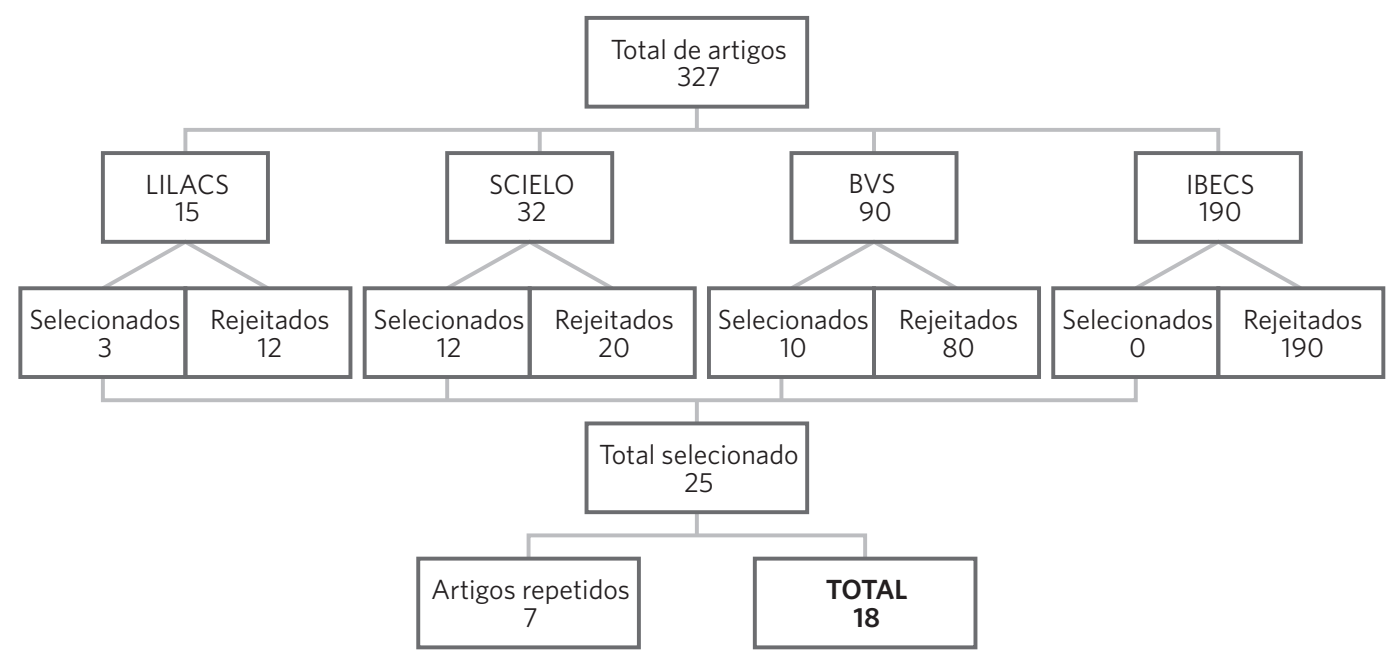

Pode-se observar, por meio da figura 1, que, entre os 327 artigos encontrados, aqueles que atenderam aos critérios de inclusão foram em número de 25 , e destes, em razão da repetitividade nas bases de dados, 18 resultaram do processo de filtragem.

Com o propósito de registrar e organizar os achados, foi criado um quadro composto pelas informações obtidas nos 18 artigos encontrados, as quais foram agrupadas nas categorias: título, autores, ano, periódico, indicador de estratificação da qualidade da produção Qualis-Capes, filiação institucional, objetivo, tipo de estudo, idioma e principais resultados. Dessa forma, foi possível analisar a composição dos estudos. Em seguida, cada artigo foi lido na íntegra, onde os aspectos mais relevantes para responder aos objetivos da pesquisa foram selecionados e organizados. Dessa maneira, foram criadas as categorias e subcategorias para exposição dos resultados, apresentados a seguir.

\section{Resultados e discussão}

\section{Idioma, autores e universidades as- sociados à produção científica sobre PICs}

Todos os artigos encontrados foram publicados no idioma português. O quadro 1 possibilita visualizar os autores e as universidades responsáveis pelas publicações. 
Quadro 1. Distribuição de autores e respectivas filiações acadêmicas

\begin{tabular}{|c|c|c|c|}
\hline № & $\begin{array}{l}\text { № de } \\
\text { Autores }\end{array}$ & Autores & Filiação Acadêmica \\
\hline 01 & 02 & $\begin{array}{l}\text { - Maria Valquíria Nogueira do Nascimento (I) } \\
\text { - Isabel Fernandes de Oliveira (II) }\end{array}$ & $\begin{array}{l}\text { (I) - Universidade Federal de Campina Grande } \\
\text { (II) - Universidade Federal do Rio Grande do Norte }\end{array}$ \\
\hline 02 & 05 & $\begin{array}{l}\text { - Christiane Gasparini Araújo Costa } \\
\text { - Mariana Tarricone Garcia } \\
\text { - Silvana Maria Ribeiro } \\
\text { - Marcia Fernanda de Sousa Salandini } \\
\text { - Cláudia Maria Bógus }\end{array}$ & Universidade de São Paulo \\
\hline 03 & 02 & $\begin{array}{l}\text { - Danielle Sousa Silva Varela (I) } \\
\text { - Dulcian Medeiros de Azevedo (II) }\end{array}$ & $\begin{array}{l}\text { (I) - Universidade Federal do Piauí } \\
\text { (II) - Universidade do Estado do Rio Grande do Norte }\end{array}$ \\
\hline 04 & 03 & $\begin{array}{l}\text { - Karla Morais Seabra Vieira Lima (I) } \\
\text { - Kênia Lara Silva (I) } \\
\text { - Charles Dalcanale Tesser (II) }\end{array}$ & $\begin{array}{l}\text { (I) - Universidade Federal de Minas Gerais } \\
\text { (II) - Universidade Federal de Santa Catarina }\end{array}$ \\
\hline 05 & 02 & $\begin{array}{l}\text { - Mariana Gonzalez Martins de Magalhães } \\
\text { - Neide Aparecida Titonelli Alvim }\end{array}$ & Universidade Federal do Rio de Janeiro \\
\hline 06 & 02 & $\begin{array}{l}\text { - Cristina dos Santos Padilha } \\
\text { - Walter Ferreira de Oliveira }\end{array}$ & Universidade Federal de Santa Catarina \\
\hline 07 & 03 & $\begin{array}{l}\text { - Wania Maria Papile Galhardi } \\
\text { - Nelson Filice de Barros } \\
\text { - Ana Cláudia Moraes Barros Leite-Mor }\end{array}$ & Universidade Estadual de Campinas \\
\hline 08 & 03 & $\begin{array}{l}\text { - Dayane Cordeiro Machado } \\
\text { - Silvia Beatriz Costa Czermainski } \\
\text { - Edyane Cardoso Lopes }\end{array}$ & Universidade Federal do Rio Grande do Sul \\
\hline 09 & 05 & $\begin{array}{l}\text { - Islândia Maria Carvalho de Sousa (I), (II) } \\
\text { - Regina Cele de Andrade Bodstein (II) } \\
\text { - Charles Dalcanale Tesser (III) } \\
\text { - Francisco de Assis da Silva Santos (I) } \\
\text { - Virginia Alonso Hortale (II) }\end{array}$ & $\begin{array}{l}\text { (I) - Fundação Oswaldo Cruz - Recife } \\
\text { (II) - Fundação Oswaldo Cruz - Rio de Janeiro } \\
\text { (III) - Universidade Federal de Santa Catarina }\end{array}$ \\
\hline 10 & 02 & $\begin{array}{l}\text { - Sônia de Castro S Thiago } \\
\text { - Charles Dalcanale Tesser }\end{array}$ & Universidade Federal de Santa Catarina \\
\hline 11 & 06 & $\begin{array}{l}\text { - João Felício Rodrigues-Neto } \\
\text { - Leonardo Santos Lima } \\
\text { - Lucas Ferreira Rocha } \\
\text { - Juliano Santos Lima } \\
\text { - Kênia Rabelo Santana } \\
\text { - Marise Fagundes Silveira }\end{array}$ & Universidade Estadual de Montes Claros \\
\hline 12 & 02 & $\begin{array}{l}\text { - Melissa Costa Santos } \\
\text { - Charles Dalcanale Tesser }\end{array}$ & Universidade Federal de Santa Catarina \\
\hline 13 & 05 & $\begin{array}{l}\text { - Luciene Alves Moreira Marques (I) } \\
\text { - Flávia Vanessa Vieira Ribeiro do Vale (I) } \\
\text { - Valéria Aparecida dos Santos Nogueira (II) } \\
\text { - Fábio Luiz Mialhell } \\
\text { - Lara Cristina Silva (I) }\end{array}$ & $\begin{array}{l}\text { (I) - Universidade Federal de Alfenas } \\
\text { (II) - Universidade Estadual de Campinas }\end{array}$ \\
\hline 14 & 04 & $\begin{array}{l}\text { - João Felício Rodrigues-Neto } \\
\text { - Maria Fernanda Santos Figueiredo } \\
\text { - Anderson Antônio de Silveira Faria } \\
\text { - Marise Fagundes }\end{array}$ & Universidade Estadual de Montes Claros \\
\hline
\end{tabular}


Quadro 1. (cont.)

\begin{tabular}{|c|c|c|c|}
\hline № & $\begin{array}{l}\text { № de } \\
\text { Autores }\end{array}$ & Autores & Filiação Acadêmica \\
\hline 15 & 06 & $\begin{array}{l}\text { - Rosália Garcia Neves (I) } \\
\text { - Leandro Barbosa de Pinho (II) } \\
\text { - Roxana Isabel Cardozo Gonzáles (I) } \\
\text { - Jenifer Harter (II) } \\
\text { - Jacó Fernando Schneider (II) } \\
\text { - Annie Jeanninne Bisso Lacchini (II) }\end{array}$ & $\begin{array}{l}\text { (I) - Universidade Federal de Pelotas } \\
\text { (II) - Universidade Federal do Rio Grande do Sul }\end{array}$ \\
\hline 16 & 02 & $\begin{array}{l}\text { - Silvana Cappelleti Nagai (I) } \\
\text { - Marcos de Souza Queiroz (II) }\end{array}$ & $\begin{array}{l}\text { (I) - Universidade Paulista } \\
\text { (II) - Universidade Estadual de Campinas }\end{array}$ \\
\hline 17 & 04 & $\begin{array}{l}\text { - Thatianny Tanferri de Brito Paranaguá } \\
\text { - Ana Lúcia Queiroz Bezerra } \\
\text { - Marcus Antônio de Souza } \\
\text { - Karina Machado Siqueira }\end{array}$ & Universidade Federal de Goiás \\
\hline 18 & 04 & $\begin{array}{l}\text { - Fabrício Fontanella } \\
\text { - Frederico Pires Speck } \\
\text { - Anna Paula Piovezan } \\
\text { - Irene Clemes Kulkamp }\end{array}$ & Universidade do Sul de Santa Catarina \\
\hline
\end{tabular}

Por meio do quadro 1, é possível perceber que, entre os 18 artigos, há 55 diferentes autores. Entre eles, Charles Dalcanale Tesser; João Felício Rodrigues-Neto e Marise Fagundes estão presentes em mais de um estudo. Neste cenário, destaca-se Charles Dalcanale Tesser, com autoria de quatro artigos.

Outro aspecto analisado é que quatro universidades acolhem pesquisadores que têm mais de um artigo publicado, sendo elas: Universidade Federal do Rio Grande do Sul (2 artigos); Universidade Estadual de Montes Claros (2 artigos); Universidade Estadual de Campinas (3 artigos); e Universidade Federal de Santa Catarina, que se destaca com 5 artigos, como origem do maior número de estudos publicados na área por pesquisadores a ela vinculados. Depreende-se que esta última se configura como um centro de estudos sobre PICs.

A respeito da quantidade de autores por publicação, entre os 18 artigos, encontraram-se sete $(38,9 \%)$ que reúnem dois autores, portanto, a maioria; três artigos reúnem cinco, quatro e três autores, respectivamente; e seis autores estão associados à produção de dois artigos.

\section{Periódicos, os anos de publicação e a classificação Qualis Capes}

O periódico 'Ciência e Saúde Coletiva' acolheu cinco artigos para publicação com o tema PICs. Observa-se, ainda, que é o único periódico que se repete entre todos os estudos analisados. Trata-se de uma revista classificada com o estrato Qualis/Capes B1 na área da saúde coletiva. Depreende-se que talvez o escopo do periódico ou o próprio nome deste seja a razão de atração, escolha ou preferência dos autores que pesquisam a temática.

No ano de 2006, foi implementada a Política Nacional que trouxe as PICs ao SUS. Nesse ano não se encontraram publicações sobre o tema. Algo que é compreensível, dado o tempo necessário à produção e publicação de um estudo. Observa-se, ainda, que nos cinco primeiros anos da implementação da PNPIC as produções foram modestas, com média de um artigo por ano.

Entre os anos de 2011 e 2014 parece ter havido maior interesse dos pesquisadores a respeito do tema PICs, sendo significativo o incremento das publicações. Nesse período, 
encontram-se 12 das 18 publicações analisadas. Destaque é dado ao ano de 2012.

O estrato Qualis/Capes B1 caracteriza a maioria dos periódicos que acolheram estudos sobre PICs.

\section{Verbos que caracterizam o objetivo dos estudos sobre PICs}

A análise empreendida a respeito dos objetivos declarados nos estudos considerou a Taxonomia de Bloom ${ }^{6}$ para identificar o domínio pretendido pelos autores. Neste sentido, encontrou-se:

(a) Quanto ao interesse dos autores em determinar, por meio do objetivo do estudo, o estágio cognitivo de 'análise' (analisar), foram encontrados 06 artigos. Os verbos utilizados para tanto foram: analisar (no objetivo de 04 artigos) e investigar (no objetivo de 01 artigo); caracterizar (no objetivo de 01 artigo).

(b) Com o intuito de determinar o estágio cognitivo de 'compreensão' (compreender), foram encontrados os verbos descrever (no objetivo de 02 artigos); identificar (no objetivo de 01 artigo) e discutir (no objetivo de 01 artigo).

(c) Os verbos que objetivam 'demonstração' (demonstrar) utilizados foram apresentar (no objetivo de 01 artigo) e focalizar (no objetivo de 01 artigo).

(d) Para determinar o estágio cognitivo de 'conhecimento', o verbo conhecer foi utilizado (no objetivo de 01 artigo).

(e) Quando a determinação do estágio cognitivo de 'avaliação' (avaliar) foi planejada, o verbo avaliar (no objetivo de 01 artigo) foi utilizado.

A partir dos achados, observa-se que a maioria dos artigos pretendeu analisar o contexto das PICs.

\section{Principais resultados apresentados pelos autores}

Para análise dos dados encontrados nos artigos, as conclusões foram organizadas em categorias: 1) O que são PICs; 2) Tipos de PICs utilizadas; 3) Principais resultados decorrentes da utilização de PICs; 4) Principais problemas/ doenças tratados com PICs; 5) Potencialidades e êxitos na implementação de PICs na atenção básica; 6) Dificuldades/fragilidades encontradas na implementação das PICs na atenção básica; 7) Percepção dos usuários/profissionais sobre PICs; 8) Mobilização, autonomia e controle social.

\section{O que são PICs}

As PICs trazem uma perspectiva holística e podem ser ferramentas para promover saúde, pois ressignificam o processo saúde-doença e propõem maior empoderamento do usuário ${ }^{7}$. Outros estudos também apontam as PICs como práticas promotoras de saúde 8 . Ainda nesse âmbito, observa-se que o modelo de assistência complementar consiste em uma postura mais abrangente, que vai além dos procedimentos médicos comuns, pois ultrapassa os aspectos físicos e considera as questões sociais, culturais e emocionais, $o$ que preceitua espaço para uma perspectiva multidisciplinar?.

As PICs também são descritas como meio para efetivar um dos princípios do SUS: a integralidade. Para que isso seja possível, é necessário que haja um cuidado especial acerca da sua implementação, pois essas práticas devem expressar a integralidade da assistência, e não se tornar apenas mais uma prestação de serviço baseada nas mesmas atitudes da biomedicina' ${ }^{1}$. Além disso, as PICs podem proporcionar uma assistência humanizada, segura, eficaz e universal, como suporte para a Medicina ${ }^{10}$.

É possível considerar que o sucesso da implementação das PICs no SUS pode ser influenciado pelo descontentamento da 
população com serviços de saúde já disponíveis. Quando os usuários têm possibilidade de acessar um serviço complementar, que vai além da medicina convencional, podem se sentir mais satisfeitos, com suas necessidades integralmente acolhidas ${ }^{\mathbf{1 1}}$. Ainda, é importante considerar que a alopatia representa problemas ao SUS, seja pela dificuldade de acesso ou pelo alto custo. Além disso, em muitos casos, os medicamentos têm sua eficácia limitada, ou comportam efeitos adversos ${ }^{\mathbf{1 2}}$.

Além das práticas individuais, existem os recursos coletivos, que podem favorecer abordagens mais complexas e abrir espaços para discussões, socialização e quebra de paradigmas, construindo saúde com participação dos usuários ${ }^{13}$.

\section{Tipos de PICs utilizadas}

Nos artigos analisados, foram citadas as PICs: fitoterapia; homeopatia; acupuntura; terapia de florais; meditação; yoga; terapia comunitária e biodança. Destacaram-se as práticas de fitoterapia (cinco citações), homeopatia (cinco citações) e acupuntura (três citações) como as mais referidas nos estudos.

Também estiveram presentes práticas que não se enquadram na PNPIC, mas que também são utilizadas como práticas complementares em saúde: relaxamento; Tai Chi Chuan; hortas comunitárias; atividades da dinâmica energética do psiquismo; teatro do oprimido; oficina de memória; dança sênior ${ }^{8,7,13,14}$.

As atividades grupais foram citadas como acompanhamento complementar à saúde: grupos de suporte mútuo; cuidadores de Alzheimer; tenda do conto; grupo de prosa com mulheres; grupo de bordadeiras; grupo de idosos; grupo de caminhadas; grupo de terapia e arte; grupos de contação de histórias ${ }^{7,13}$. A oração a Deus e a busca de benzedeiras também são apontadas como estratégias popularmente utilizadas para o autocuidado, também como complemento da assistência em saúde ${ }^{15}$.

\section{Principais resultados decorrentes da utilização de PICs}

Os estudos revelam: redução da medicalização ${ }^{13}$, empoderamento dos usuários na busca do autocuidado e a responsabilização pela própria saúde ${ }^{7}$; possibilidade de reduzir a frequência de transtornos mentais comuns ${ }^{11}$; autonomia e participação do usuário na escolha das suas estratégias de tratamento'; e "baixo custo, ausência de efeitos colaterais, satisfação e crença da população"10(1).

Em pesquisa realizada no estado de Minas Gerais ${ }^{\mathbf{1 6}}$ com usuários do serviço de saúde pública, observa-se que a maioria dos entrevistados aceitaria o uso de PICs, caso fossem ofertadas. Outro estudo identificou que há aceitação dos profissionais quanto à implementação das PICs na rede pública de saúde, mesmo que haja uma pequena parcela de profissionais que expressam desconfiança acerca dessa proposta9.

Os estudos atuais sustentam a hipótese de que existe um crescente interesse pelas PICs, tanto por parte dos profissionais quanto dos usuários, que têm buscado esse modelo de assistência complementar ${ }^{17}$. Observa-se que a busca dos profissionais por capacitação nessa área e a procura dos pacientes pelo serviço podem representar uma significativa mudança cultural acerca da assistência em saúde. Inclusive, muitas pessoas buscam essas práticas mesmo sem recomendação profissional ${ }^{12}$.

A aceitação das PICs por parte dos profissionais de saúde também pode ter relação com a formação e o papel exercido por cada um deles. Os enfermeiros teriam maior abertura à utilização de PICs do que os médicos. Citam que os médicos justificam sua prática dentro da própria biomedicina e da medicalização. Por outro lado, os profissionais de enfermagem teriam maior interesse em ofertar estratégias não medicamentosas ${ }^{17}$.

Identificou-se em pesquisa que a maior parte dos gestores e dos profissionais de saúde não conhecia a PNPIC. Por sua vez, os profissionais que a conheciam a utilizavam para 
disseminar e justificar a utilização de homeopatia ${ }^{18}$. Outro estudo observou a percepção dos gestores sobre fitoterapia e outras PICs, identificando que no município pesquisado há uma boa perspectiva de implementação das PICs na atenção básica. Ainda se viu que a prescrição de plantas medicinais é maior do que a de fitoterápicos ${ }^{19}$.

A PNPIC trouxe ao SUS abordagens que anteriormente eram disponíveis especialmente por meio de prestadores privados. Autores relatam que práticas como acupuntura e homeopatia ainda são mais predominantes no serviço privado, todavia, apontam que a acupuntura se dissemina no SUS após ser exercida por outros profissionais que não apenas os médicos ${ }^{\mathbf{1 4}}$.

Existem algumas atividades que não são ofertadas no serviço privado, como as hortas comunitárias. Essa prática se adequa à proposta de promoção de saúde no SUS. As entrevistas realizadas por pesquisadores revelam resultados positivos na saúde mental das pessoas envolvidas nas atividades de contato com a terra ${ }^{8}$. O trabalho coletivo fortalece os vínculos sociais e a participação na comunidade, valoriza a história e os conhecimentos populares. As hortas comunitárias oportunizam o fortalecimento do uso de plantas medicinais, que, por consequência, traz a redução da alopatia ${ }^{8}$.

Em Montes Claros (MG), práticas complementares e alternativas são amplamente utilizadas por pessoas infectadas pelo Vírus da Imunodeficiência Humana (HIV) que realizam tratamento em um centro de referência de doenças sexualmente transmissíveis. Entre as práticas referidas pelos pacientes, a principal delas é a oração a Deus, de forma terapêutica, além dos populares 'benzimentos'. Acerca dos transtornos mentais, os autores revelam uma predominância no uso de homeopáticos, sugerindo que esse dado se refere ao maior tempo de reconhecimento dessa prática pelo Conselho Federal de Medicina, se comparada com outras PICs ${ }^{15}$.

Estudiosos trazem uma perspectiva importante sobre a implementação das PICs como estratégia para a assistência em saúde, sugerindo que tais práticas não estão em oposição à Medicina, mas complementando-a e até mesmo transcendendo-a. Citam, ainda, que a implementação das PICs na atenção básica está de acordo com o ideal do SUS e com as recomendações da Organização Mundial da Saúde. Nesse mesmo estudo, os profissionais referiram que muitos dos problemas pelos quais as pessoas buscam o serviço de saúde não têm sua resolutividade garantida ou possível por meio da alopatia. Concluem os autores que, dessa forma, as PICs viriam a preencher essa lacuna?.

\section{Principais problemas/doenças trata- dos com PICs}

Um estudo destaca que as principais demandas referidas pelos usuários de PICs são: transtornos mentais graves e leves; questões familiares, laborais, sociais e econômicas; sintomas psicossomáticos diversos; alternativa a medicalização; hipertensão, diabetes e outras doenças crônicas ${ }^{13}$.

Outros estudos também referem questões como ansiedade, insônia e transtornos mentais comuns como principais problemas tratados com apoio das PICs na atenção básica ${ }^{11,20}$. Enfatizase o uso de fitoterápicos como coadjuvantes para prevenção e controle de doenças como a hipertensão, onde, por exemplo, uma situação de ansiedade poderia resultar no aumento da pressão arterial, e, dessa forma, o uso da terapia complementar poderia apoiar o medicamento anti-hipertensivo e evitar agravos ${ }^{20}$.

Foi observada a prevalência do uso de fitoterápicos com propriedades ansiolíticas e sedativas no tratamento de problemas como menopausa e outros de ordem ginecológica ${ }^{20}$.

\section{Potencialidades, êxitos e desafios na implementação de PICs na atenção básica}

É cada vez maior a multidisciplinaridade na realização de PICs grupais. Autores destacam a ampla participação de Agentes Comunitários de 
Saúde (ACSs) nesse contexto, sendo profissionais de grande potencialidade, pois são membros tanto da equipe quanto da comunidade ${ }^{13}$.

Muitas das PICs são inseridas na prática pelos profissionais que acreditam em formas diferentes de assistência e que buscam melhoras nos serviços ${ }^{13}$.

É evidente a importância de abordar PICs nas graduações na área da saúde, de forma que os profissionais recém-formados já adentrem a prática profissional inteirados dessas abordagens e sua aplicabilidade ${ }^{20}$. No Brasil, algumas universidades federais ofertam disciplinas eletivas de homeopatia e acupuntura nos cursos de Medicina ${ }^{14}$.

Faz-se necessário ampliar os recursos para atividades de promoção de saúde, assim como ofertar suporte técnico para elas ${ }^{8}$. A educação permanente é uma importante estratégia para incorporar as PICs na atenção básica e repensar a centralidade dos médicos e da medicação como únicos responsáveis por buscar solucionar problemas de saúde. Supõe-se que o oferta das PICs no sistema de saúde está associada ao apoio da gestão e ao interesse dos profissionais ${ }^{\mathbf{1 4}}$. A divulgação da PNPIC é importante, tanto para profissionais quanto para usuários dos serviços ${ }^{16}$. Também são pertinentes pesquisas científicas nessa área, para que se possa examinar as potencialidades, os benefícios e limites das práticas ${ }^{21}$.

Para estabelecer as PICs na atenção básica, é necessário considerar todo o processo que envolve política, gestão, recurso humano, cultura do local, cultura da organização do trabalho, recursos disponíveis, entre outros. A colaboração participativa na consolidação das PICs é importante, pois subsidia a mudança de pensamento e a consequente mudança de cultura ${ }^{22}$. A implementação bem-sucedida das PICs tem relação com quatro pontos principais: a disposição dos usuários em receber esses serviços; a percepção de médicos sanitaristas sobre saúde e sua abertura para as práticas complementares; apoio das categorias profissionais que pretendem ampliar suas possibilidades de intervenção; a ideologia contida nas PICs, que condiz com a integralidade proposta no SUS 9 .

Além da inserção das PICs diretamente nas Unidades Básicas de Saúde, outra estratégia utilizada para ofertar as práticas no SUS é a criação de centros especializados nesse serviço ${ }^{7}$.

É necessário atentar para que a essência da PNPIC não se perca, que esse modelo complementar de assistência não se torne mais uma prestação de serviço mecanicista e técnica, sem considerar toda a complexidade de fatores envolvida na saúde'.

Sugere-se capacitar os ACSs para informar a população sobre a importância das PICs, ampliando as possibilidades de autocuidado e trazendo mais alternativas para promoção de saúde. Para os autores, não apenas os ACSs, mas todos os profissionais da saúde devem ser incentivados a ampliar sua perspectiva a respeito da saúde, com a finalidade de compreender que o modelo biomédico pode não ser capaz de isoladamente solucionar uma diversidade de problemas ${ }^{\mathbf{1 0}}$. As PICs já fazem parte do SUS, dessa forma, o próximo passo é trazê-las para perto dos profissionais, inserindo-as em seus contextos de atuação ${ }^{21}$. É, portanto, um desafio sensibilizar usuários e profissionais, assim como capacitar as equipes para o uso das práticas ${ }^{8}$.

A implementação das PICs na atenção básica representa a adesão de uma postura mais integral e abrangente, que vai além da biomedicina e considera o sujeito em sua totalidade emocional, social e cultural, adotando uma abordagem multidisciplinar ${ }^{9}$.

\section{Dificuldades e fragilidades referidas quanto à implementação das PICs na atenção básica}

Apesar de as PICs fazerem parte do serviço de saúde, estão às margens dele. Pesquisadores se referem à falta de apoio da gestão e das instituições que formam profissionais. A carência de recursos materiais, de infraestrutura e a fragilidade do trabalho em equipe afetam significativamente a implementação 
das PICs ${ }^{13}$. Foram identificados dois aspectos negativos da implantação na atenção básica, sendo eles o pouco planejamento e uma noção simplista do que são as PICs, e o entendimento errôneo sobre doença. $\mathrm{O}$ estudo também sugere a criação de uma coordenação de PICs, que seja capaz de planejar adequadamente a gestão das práticas?.

Considera-se que a PNPIC favoreceu o registro das práticas nos sistemas de informação, entretanto, não há uma definição exata do que pode ser registrado como PIC. Essa indefinição pode apresentar impasses para a inserção das práticas no serviço de saúde ${ }^{7}$. Outros autores reforçam esse entendimento, expondo que as Portarias $n^{\circ}$ 971, de 2006, e $\mathrm{n}^{\mathrm{o}} 853$, de 2006, são pouco esclarecedoras sobre o que pode ser registrado como prática corporal e outras técnicas, o que torna os registros pouco específicos. A mensuração e a avaliação ainda constituem um desafio, pois existe discrepância entre o que é produzido e o que é registrado nos sistemas de informação ${ }^{\mathbf{1 4}}$.

A atuação profissional exerce significativo impacto quando o assunto é dificuldade na implementação das PICs. Destaca-se que os cursos de graduação nem sempre preparam os acadêmicos de Medicina para prescrição de fitoterápicos, sendo assim, eles já vêm para a prática com esse déficit formativo ${ }^{20}$. Uma investigação possibilitou identificar que muitos profissionais não compreendem a perspectiva da promoção de saúde relacionada às PICs, confundindo o conceito com o da prevenção de agravos ${ }^{7}$.

Nota-se que há desconhecimento dos profissionais quanto ao tema, o que revela necessidade de capacitação e maior divulgação do assunto ${ }^{17}$. Segundo estudo, os benefícios dessas terapias devem ser mais divulgados, especialmente aos médicos ${ }^{16}$. Sobre isso, considera-se que a ciência médica ainda apresenta desconfiança sobre as $\mathrm{PICs}{ }^{\mathbf{2 1}}$. Autores citam que muitos pacientes não informam seus médicos sobre a utilização de medicinas complementares e alternativas, por receio de comprometer o tratamento realizado ${ }^{\mathbf{1 2}}$.
Sobre a capacitação em PICs, observa-se que a maioria dos cursos é ofertada por instituições de cunho privado, dessa forma, a ausência de investimento na formação de profissionais para as PICs dificulta o aumento da sua oferta na saúde pública ${ }^{\mathbf{1 4}}$.

Por outro lado, os profissionais que possuem formação na área de PICs nem sempre têm acesso a espaço físico e materiais para executar suas ações; muitas vezes, não possuem nem autorização para fazê-las. Resulta disso que tais profissionais acabam por transferir suas práticas ao serviço privado ${ }^{17}$. As práticas estão sendo difundidas e amplamente utilizadas, mas se tornam de difícil acesso em razão do alto custo ${ }^{10}$.

Observam-se fragilidades nas PICs em atividades grupais, pois muitas vezes não estão previstas como parte do serviço de saúde. Dessa maneira, podem aparentar um trabalho invisível ou menos importante que os demais. Além disso, a relação verticalizada entre o profissional e a comunidade pode dificultar as ações coletivas ${ }^{\mathbf{1 3}}$. Muitas vezes, os profissionais da atenção básica apresentam uma postura menos horizontalizada do que os de atenção secundária, como é o caso dos Centros de Atenção Psicossocial23. Outras fragilidades na efetivação das atividades coletivas são: falta de sensibilização dos médicos para a importância da recomendação dessas atividades aos seus pacientes; falta de adesão de profissionais na execução das ações; falta de capacitação da equipe para exercer educação em saúde ${ }^{8}$.

\section{Percepção dos usuários/profissionais sobre PICs}

É possível perceber nos resultados dos artigos objeto desta pesquisa que as atividades coletivas ofertadas pelas equipes de saúde são percebidas positivamente pela comunidade. A horta comunitária é descrita como uma atividade terapêutica que envolve participação efetiva das pessoas em um espaço saudável, de bem-estar, onde ocorre integração entre comunidade e serviço de saúde ${ }^{8}$. 
A terapia comunitária aparece na produção pesquisada como outra forma de estabelecer e fortalecer vínculos, além de incentivar a autonomia da comunidade. O terapeuta comunitário, apesar de orientar o andamento do grupo, não está em uma posição de superioridade ou de detenção do conhecimento, mas assume uma posição de igualdade com os demais. Nesse sentido, o terapeuta comunitário é percebido como "alguém que humaniza as relações interpessoais no setor saúde"23(1).

Os artigos analisados possibilitam constatar que tanto os profissionais quanto os usuários buscam as PICs como forma de melhorar a saúde ${ }^{19}$. No caso dos profissionais, as residências e demais especializações na área de saúde da família e comunidade podem estar relacionadas à boa aceitação das PICs por eles ${ }^{17}$.

Encontra-se, ainda, que a insatisfação com o modelo biomédico, com seus métodos invasivos, com altos custos, foco na doença e impessoalidade, é uma motivação significativa para o interesse pelas PICs ${ }^{17}$.

\section{Mobilização, autonomia e controle social}

Por meio das análises empreendidas nos artigos selecionados, observa-se que o trabalho coletivo representa um significativo potencial na atenção básica. Os grupos são contextos favoráveis para produzir saúde ${ }^{13}$. Ao reunir um grupo, tem-se potencial de realizar construções coletivas, onde se pode discutir sobre saúde, autocuidado, alimentação, entre outros. Esses encontros podem proporcionar bem-estar, pois o sujeito encontra um tempo para si, reflete sobre a própria saúde, à medida que se relaciona com o meio social. A integração e o envolvimento na atividade coletiva criam sentimento de pertencimento e fazem o usuário se aproximar da equipe de saúde 8 .

Foi observado nos artigos analisados que usuários de terapia floral se apresentaram mais participativos no seu próprio cuidado e que estabelecem uma relação mais horizontal com o profissional. Segundo os autores, essa relação ocorre através do diálogo e da possibilidade de escolha, algo que difere do assistencialismo'. Ainda nesse sentido, a humanização deve estar orientada por

autonomia, corresponsabilidade, protagonismo dos sujeitos envolvidos, solidariedade entre os vínculos estabelecidos, respeito aos direitos dos usuários e participação coletiva no processo de gestão1(652).

A terapia comunitária aparece nos artigos como uma abordagem que por si só expressa o mais puro significado de autonomia e participação, onde os usuários, por meio de sua relação com outros membros da comunidade, estabelecem vínculos de suporte mútuo ${ }^{23}$.

A horta comunitária, conforme referem os autores dos artigos, representa uma técnica rica para abordar saúde no âmbito integral. Para os autores, o cultivo de plantas medicinais e alimentos orgânicos por si só é uma atividade promotora de saúde, que origina espaços para a criação de hábitos saudáveis e capacitação da comunidade para fazer uso desses recursos. Prosseguem afirmando que o trabalho conjunto e o estreitamento das relações entre Unidade Básica de Saúde e a população também são importantes para efetivação das propostas da atenção básica e da PNPIC ${ }^{8}$.

Encontra-se nos artigos, ainda, que a horta propicia busca de conhecimentos, reflexão sobre preservação dos recursos naturais, combate ao desperdício, alimentação e os hábitos saudáveis, além da participação e do controle social. Diversas demandas podem ser trabalhadas nesse meio, tanto no âmbito da saúde física quanto mental e social ${ }^{8}$.

\section{Considerações finais}

O presente estudo buscou conhecer as características da produção brasileira sobre PICs na atenção básica, identificar as tendências dessas pesquisas, registrar seus principais resultados e avaliar as características da utilização das práticas. 
Considera-se que profissionais e usuários buscam nas PICs possibilidades de melhoria da saúde e da qualidade de vida. Nesse sentido, a insatisfação de muitos usuários com o modelo biomédico pode ampliar o interesse pelas PICs, como suporte para a assistência em saúde. A autonomia dos usuários em optar pelos tratamentos complementares os faz sentir protagonistas e corresponsáveis pelo próprio cuidado. Nesse aspecto, as atividades grupais são percebidas positivamente, em razão de representarem contextos favoráveis à produção de saúde, compartilhamento e participação social, assim como de fortalecimento da relação horizontal usuário-serviço.

Assim entende-se que as pesquisas brasileiras acerca das PICs tendem a crescer conforme se mostram os êxitos dessas práticas na saúde pública. Os estudos qualitativos têm destaque por avaliarem as questões subjetivas resultantes da oferta desses serviços, tendo em vista que as PICs favorecem a saúde mental e despertam o autocuidado. Percebese através dos estudos que, mesmo havendo uma política nacional estabelecida, as PICs, em muitos contextos, são implementadas de maneira independente pelos próprios profissionais interessados, frequentemente não havendo planejamento da gestão nem recursos disponíveis para sua realização. Por esse motivo, os estudos na área de planejamento, implementação e gestão das PICs podem ser tendência para os próximos anos, apoiando o crescimento e a qualidade da oferta.

A formação dos profissionais para aplicação e fomento das PICs no SUS deve receber especial atenção, pois, mesmo que não se trate de procedimentos invasivos ou de alto risco, devem ser administrados com responsabilidade, compreendendo as potencialidades e os limites oferecidos por essas terapias, que precisam ser incluídas nos serviços com o devido planejamento e objetivos claros, de forma a evitar a banalização ou o mau uso desses tratamentos, os quais são tão sérios quanto os demais.

Conclui-se, com base nos resultados dos artigos publicados entre 2006 a 2016, que as PICs não devem ser vistas como uma estratégia para reparar ou substituir os elementos do sistema que não funcionam de maneira satisfatória, visto que elas próprias possuem diversas limitações. Essas práticas se apresentam no SUS como complemento a uma assistência em saúde que já exerça bom funcionamento, dessa forma, podem vir a contribuir para complemento e melhoramento de uma assistência já efetiva, oferecendo estratégias de autocuidado, promoção de saúde e qualidade de vida. Os artigos analisados também levam a crer que as PICs têm amplo potencial de melhoramento dos serviços de saúde, não apenas da atenção básica, mas também em outras instâncias.

\section{Colaboradores}

Aguiar J (0000-0002-4612-3968)*, Kanan LA (0000-0001-6412-0544)* e Masiero AV (00000002-2611-4108)* contribuíram igualmente para a elaboração do manuscrito. 


\section{Referências}

1. Magalhães MGM, Alvim NAT. Práticas integrativas e complementares no cuidado de enfermagem: um enfoque ético. Esc. Anna Nery. [internet]. 2013 [acesso em 2018 mar 21]; 17(4):646-653. Disponível em: http://www.scielo.br/scielo.php?script=sci arttext\&pid=S1414-81452013000400646\&lng=en\&n $\mathrm{rm}=\mathrm{iso}$.

2. Telesi Junior E. Práticas integrativas e complementares em saúde, uma nova eficácia para o SUS. Estud. Av. [internet] 2016 [acesso em 2017 nov 4]; 30(86):99-112. Disponível em: http:// www.scielo.br/scielo.php?script=sci_arttext\&pid =S0103-40142016000100099.

3. Brasil. Ministério da Saúde. Política Nacional de Práticas Integrativas e Complementares no SUS. Brasília, DF: Ministério da Saúde; 2006.

4. Minas Gerais. Secretaria de Estado de Saúde. Política Nacional de Práticas Integrativas e Complementares no SUS - PNPIC-SUS. [acesso em 2020 fev 11]. Disponível em: http://www.saude.mg.gov.br/pics.

5. Araújo CA. Bibliometria: evolução histórica e questões atuais. Em Questão. [internet] 2006 [acesso em 2017 nov 4]; 12(1):11-32. Disponível em http://www. redalyc.org/html/4656/465645954002/.

6. Ferraz APCM, Belhot RV. Taxonomia de Bloom: revisão teórica e apresentação das adequações do instrumento para definição de objetivos instrucionais. Gest. Prod. [internet] 2010; 17(2):421-431.

7. Lima KMSV, Silva KL, Tesser CD. Práticas integrativas e complementares e relação com promoção da saúde: experiência de um serviço municipal de saúde. Interface Botucatu [internet]. 2014 [acesso em 2017 out 21]; 18(49):261-272. Disponível em: http://www. scielo.br/scielo.php?script=sci_arttext\&pid=S1414$-32832014000200261 \& \operatorname{lng}=\mathrm{en} \& \mathrm{nrm}=$ iso.

8. Costa CGA, Garcia MT, Ribeiro SM, et al. Hortas comunitárias como atividade promotora de saúde: uma experiência em Unidades Básicas de Saúde. Ci- ênc. Saúde Colet. [internet]. 2015 [acesso em 19 mar 19]; 20(10):3099-3110. Disponível em: <http://www. scielo.br/scielo.php?script=sci_arttext $\&$ pid=S1413$-81232015001003099 \& \operatorname{lng}=\mathrm{en} \& \mathrm{nrm}=\mathrm{iso}>$.

9. Nagai SC, Queiroz MS. Medicina complementar e alternativa na rede básica de serviços de saúde: uma aproximação qualitativa. Ciênc. Saúde Colet. [internet]. 2011 [acesso em 2018 maio 16]; 16(3):1793-1800. Disponível em: <http://www.scielo.br/scielo.php?script=sci_arttext\&pid=S1413$-81232011000300015 \& \operatorname{lng}=$ en\&nrm=iso $>$.

10. Paranaguá TTB, Bezerra ALQ, Souza MA, et al. As práticas integrativas na Estratégia Saúde da Família: visão dos agentes comunitários de saúde. Rev. Enferm. UERJ [internet] 2009 [acesso em $2018 \mathrm{abr}$ 21]; 17(1):75-80. Disponível em: http://www.facenf. uerj.br/v17nl/vl7nlal4.pdf.

11. Rodrigues-Neto JF, Figueiredo MFS, Faria AAS, et al. Transtornos mentais comuns e o uso de práticas de medicina complementar e alternativa: estudo de base populacional. J Bras. Psiquiatr. [internet]. 2008 [acesso em 2018 maio 7]; 57(4):233-239. Disponível em: <http://www.scielo.br/scielo.php?script=sci arttext\&pid=S0047-20852008000400002\&lng=en $\&$ nrm=iso>.

12. Fontanella F, Speck FP, Piovezan AP, et al. Conhecimento, acesso e aceitação das práticas integrativas e complementares em saúde por uma comunidade usuária do Sistema Único de Saúde na cidade de Tubarão/SC. Arquivos Catarinenses de Medicina [internet]. 2007 [acesso em 2018 abr 21]; 36(2). Disponível em: http://bases.bireme.br/cgi-bin/wxislind. exe/iah/online/?IsisScript=iah/iah.xis\&src=google \&base $=$ LILACS\&lang $=p \&$ nextAction $=1$ nk\&exprSea rch=464650\&indexSearch=ID

13. Nascimento MVN, Oliveira IF. As práticas integrativas e complementares grupais e sua inserção nos serviços de saúde da atenção básica. Estud. Psicol. (Natal) [internet]. 2016 [acesso em 2018 mar 14]; 21(3):272281. Disponível em: http://www.scielo.br/scielo. 
php?script=sci_arttext\&pid=S1413-294X20160003 00272\&lng=en\&nrm=iso.

14. Sousa IMC, Bodstein RCA, Tesser CD, et al. Práticas integrativas e complementares: oferta e produção de atendimentos no SUS e em municípios selecionados. Cad. Saúde Pública [internet]. 2012 [acesso em 2019 mar 21]; 28(11):2143-2154. Disponível em: http://www. scielo.br/scielo.php?script=sci_arttext\&pid=S0102$-311 X 2012001100014 \& \operatorname{lng}=\mathrm{en} \& \mathrm{nrm}=$ iso.

15. Rodrigues-Neto JF, Lima LS, Rocha LF, et al. Uso de práticas integrativas e complementares (PIC) por pacientes adultos infectados com o vírus da imunodeficiência humana (HIV), no norte de Minas Gerais. Rev. Baiana Saúde Pub. [internet]. 2010 [acesso em 2019 abr 28]; 34(1):159-172. Disponível em: http://files.bvs.br/upload/S/0100-0233/2010/v34nl/a1457. pdf.

16. Marques LAM, Vale FVVR, Nogueira VAS, et al. Atenção farmacêutica e práticas integrativas e complementares no SUS: conhecimento e aceitação por parte da população sãojoanense. Physis [internet]. 2011 [acesso em 2019 abr 30]; 21(2):663-674. Disponível em: http://www.scielo.br/scielo.php?script=sci arttext\&pid=S0103-73312011000200017\&lng=en\&nr $\mathrm{m}=$ iso.

17. Thiago SCS, Tesser CD. Percepção de médicos e enfermeiros da Estratégia de Saúde da Família sobre terapias complementares. Rev. Saúde Pública [internet]. 2011 [acesso em 2019 mar 23]; 45(2):249-257. Disponível em: http://www.scielo.br/scielo.php?script=sci arttext\&pid=S0034-89102011000200003\&lng=en\&n rm=iso.

18. Galhardi WMP, Barros NF, Leite-Mor ACMB. O conhecimento de gestores municipais de saúde sobre a Política Nacional de Prática Integrativa e Complementar e sua influência para a oferta de homeopatia no Sistema Único de Saúde local. Ciênc. Saúde Colet. [internet]. 2013 [acesso em 2018 mar 21]; 18(1):213-220. Disponível em: http://www.scie-
lo.br/scielo.php?script=sci_arttext\&pid=S1413$-81232013000100022 \& \operatorname{lng}=e n \& n r m=$ iso.

19. Machado DC, Czermainski SBC, Lopes EC. Percepções de coordenadores de unidades de saúde sobre a fitoterapia e outras práticas integrativas e complementares. Saúde debate [internet]. 2012 [acesso em 2018 mar 21]; 36(95):615-623. Disponível em: http://www. scielo.br/scielo.php?script=sci_arttext\&pid=S0103$-11042012000400013 \& \operatorname{lng}=\mathrm{en} \& \mathrm{nrm}=$ iso.

20. Varela DSS, Azevedo DM. Saberes e práticas fitoterápicas de médicos na estratégia saúde da família. Trab. Educ. Saúde. [internet]. 2014 [acesso em 2018 mar 19]; 12(2):273-290. Disponível em: http://www. scielo.br/scielo.php?script=sci_arttext\&pid=S1981$-77462014000200004 \& \operatorname{lng}=$ en\&nrm=iso.

21. Neves RG, Pinho LB, Gonzáles RIC, et al. O conhecimento dos profissionais de saúde acerca do uso de terapias complementares no contexto da atenção básica. Rev. Pesqui. Cuid. Fundam. (Online) [internet]. 2012 [acesso em 2018 maio 15]; 4(3):2502-2509. Disponível em: http://www.lume.ufrgs.br/handle/10183/140124.

22. Santos MC, Tesser CD. Um método para a implantação e promoção de acesso às Práticas Integrativas e Complementares na Atenção Primária à Saúde. Ciênc. Saúde Colet. [internet]. 2012 [acesso em 2018 mar 21]; 17(11):3011-3024. Disponível em: http://www. scielo.br/scielo.php?script=sci_arttext\&pid=S1413$-81232012001100018 \& \operatorname{lng}=\mathrm{en} \& \mathrm{nrm}=$ iso.

23. Padilha CS, Oliveira WF. Representação social do terapeuta comunitário na rede SUS. Ciênc. Saúde Colet. [internet]. 2013 [acesso em 2018 mar 21]; 18(8):2211-2220. Disponível em: http://www.scielo.br/scielo.php?script=sci_arttext\&pid=S1413$-81232013000800005 \& \operatorname{lng}=\mathrm{en} \& \mathrm{nrm}=$ iso.

Recebido em 07/03/2019

Aprovado em 09/09/2019

Conflito de interesses: inexistente

Suporte financeiro: não houve 